\title{
Changes in affect from non-eating activity engagement as predictors of loss of control eating: an ecological momentary assessment study
}

\author{
Christina R. Felonis ${ }^{1,2,3}$ - Laura Boyajian ${ }^{1,2} \cdot$ Stephanie Fan $^{1,2} \cdot$ Sarah Drexler $^{1,2} \cdot$ Brighid Fitzpatrick $^{1,2}$. \\ Adam Payne-Reichert ${ }^{1,2} \cdot$ Stephanie M. Manasse ${ }^{1,2} \cdot$ Adrienne S. Juarascio $^{1,2}$
}

Received: 5 August 2021 / Accepted: 19 November 2021 / Published online: 30 November 2021

(C) The Author(s), under exclusive licence to Springer Nature Switzerland AG 2021

\begin{abstract}
Purpose Loss of control eating (LOCE) is supported as a maladaptive regulation strategy for high negative affect (NA) and low positive affect (PA). Yet, little is known about the day-to-day activities outside of eating that may precipitate such changes in affect and impact risk for LOCE. The current ecological momentary assessment study sought to examine whether (1) low NA and high PA at a given survey would prospectively predict lower likelihood of a LOCE episode at the next survey, and (2) having recently engaged in various non-eating activities would be associated with changes in average NA and PA levels at the same survey.

Methods Data collected from 66 participants with LOCE over 7-14 days were analyzed using generalized estimating equations.

Results High NA but not low PA at a given survey prospectively predicted lower likelihood of a LOCE episode at the next survey. Having recently engaged in outdoor recreation and socializing was associated with lower than one's usual NA and higher than one's usual PA at the same survey, social media was associated with higher than one's usual NA and lower than one's usual PA, self-care was associated with higher than one's usual PA, and napping was associated with lower than one's usual PA.

Conclusion Individuals may benefit from increasing the frequency of daily non-eating activities that reduce NA, since it appears to be a more salient predictor of LOCE than PA. Further research is needed to elucidate this LOCE maintenance model, particularly in regard to the role of PA.

Level of evidence Level IV, multiple time series without intervention.
\end{abstract}

Keywords Loss of control eating $\cdot$ Negative affect $\cdot$ Positive affect $\cdot$ Non-eating activities $\cdot$ Ecological momentary assessment $\cdot$ Predictor

\section{Introduction}

Negative affect (NA) is widely recognized as a maintenance factor for loss of control eating (LOCE) [1], i.e., eating either an unambiguously large amount of food or an amount of food deemed large only by the individual combined with a

Christina R. Felonis

cf687@drexel.edu

1 Center for Weight, Eating, and Lifestyle Science, Drexel University, 3201 Chestnut St., Philadelphia, PA 19104, USA

2 Department of Psychology, Drexel University, 3201 Chestnut St., Philadelphia, PA 19104, USA

3 Drexel University, Stratton Hall Room 273, 3141 Chestnut St., Philadelphia, PA 19104, USA sense of lack of control over eating [2]. The affect regulation model conceptualizes the significant relationship between NA and LOCE in binge eating spectrum disorders $[3,4]$ as LOCE being maladaptively intended to alleviate, control, or avoid experiencing NA [1, 5-7]. Ecological momentary assessment (EMA) studies have supported this model by indicating that momentary NA is an antecedent to LOCE, such that high NA prospectively predicts the near-time occurrence of a LOCE episode [8-10].

More recently, low positive affect (PA) has also been recognized as a maintenance factor for LOCE [11, 12]. Individuals with LOCE have been found to experience lower overall and within-day levels of PA [9, 13]. Accordingly, expansions to the affect regulation model argue that individuals may also engage in LOCE to increase PA due to a 
lack of adaptive pleasurable activities in their day-to-day lives [11]. EMA studies have supported these expansions by finding that low PA precedes the near-time occurrence of a LOCE episode, and that PA stabilizes following engagement in this behavior $[9,10,14]$. These results strongly suggest that changes in both NA and PA signal risk for LOCE.

Despite the strong support for NA and PA as predictors of LOCE, less is known about the day-to-day factors that precipitate these momentary changes in affect and thus increase or decrease risk for a LOCE episode. While evidence-based treatments for LOCE such as Cognitive Behavioral Therapy and Behavioral Weight Loss have been shown to be effective at reducing the frequency of LOCE episodes, they leave substantial room for improvement with only about $50 \%$ of individuals abstaining fully at post-treatment $[15,16]$. One potential explanation for these suboptimal outcomes is that affect is minimally addressed as a maintenance factor for LOCE through a limited number of strategies such as alternative activities and stimulus control $[2,17]$. It may therefore be beneficial to identify the day-to-day factors that precipitate changes in affect to prevent these changes and proactively reduce the likelihood of a LOCE episode. Previous EMA studies that have examined the impact of such factors on affect have primarily focused on eating-related ones. For example, Stein et al. [18] found that NA and hunger were both higher prior to a LOCE episode compared to nonLOCE times. Smith et al. [19] found that when individuals had lower PA and higher biased attention toward palatable foods, believing that food would improve affect increased risk for a LOCE episode. While these studies clarify how eating-related factors impact affect and subsequently contribute to LOCE, they do not address how other day-to-day factors outside of eating may also be contributing. Research outside of the eating disorder (ED) field has found that noneating activities including cooking [20], indoor hobbies \{e.g., reading [21], digital gaming [22]\}, outdoor recreation [23, 24], socializing [25], and meditation [26, 27] improve affect, while watching TV [21], driving [28], and napping $[29,30]$ worsen or have a mixed impact on affect. However, these studies primarily utilized retrospective recall of non-eating activity engagement and affect which allows for recall error. The current study aimed to address this limitation by examining changes in affect from non-eating activity engagement in an ecologically valid manner, and by linking them to LOCE.

To date, only a few EMA studies have specifically tested how engaging in non-eating activities may impact affect and thus increase or decrease risk for a LOCE episode. Goldschmidt et al. [31] found that interpersonal stressors (e.g., an argument), work or environmental stressors (e.g., worrying about long-term goals), and general daily hassles (e.g., car trouble) prospectively predicted the near-time occurrence of LOCE, and that high NA mediated the relationship.
However, changes in NA resulting from stressful noneating activities exclusively were measured. Srivastava, Michael, Manasse, and Juarascio [32] found that spending time on social media prospectively predicted elevated body dissatisfaction which, in turn, prospectively predicted the occurrence of LOCE. However, they did not test whether this increased risk for LOCE was due to changes in affect resulting from the social media-driven elevated body dissatisfaction. Collectively, these studies describe the relationship between certain non-eating activities and maladaptive eating behaviors. However, the temporal relationship between non-eating activity engagement and changes in affect as they relate to LOCE remains unclear.

The present study thus sought to examine changes in NA and PA from engagement in non-eating activities as predictors of LOCE using an EMA protocol. As a preliminary aim, we sought to confirm that low NA and high PA at a given survey would prospectively predict lower likelihood of a LOCE episode at the next survey. As the primary aim, we sought to examine whether having recently engaged in a range of non-eating activities would be associated with changes in average NA and PA levels at the same survey.

\section{Methods}

\section{Procedure}

Participants were invited to complete an EMA protocol for 7-14 days prior to starting LOCE treatment and, if completed, were invited to also participate in the EMA study after finishing treatment. Participants received training on the EMA protocol, which included definitions of ED behaviors and completing a sample EMA survey. Every morning, participants were prompted to complete a daily survey which assessed amount of sleep, quality of sleep, current tiredness levels, engagement in risky behaviors the day prior, and impulse control. Participants were also prompted to complete five randomly spaced surveys throughout the day that assessed ED behaviors, fear of weight gain, affect, exercise engagement, shape and weight satisfaction, non-eating activity engagement, and treatment skill use. Participants were also asked to self-initiate an event-contingent survey anytime they engaged in an ED behavior such as LOCE, which assessed reasons why the ED behavior may have occurred (e.g., thoughts, feelings, and behaviors leading up to the episode, whether the episode was planned, whether participants had engaged in restrictive eating patterns since the last survey for the purposes of influencing shape and weight, and whether they had engaged in exercise). Procedures were approved and overseen by the Drexel University Institutional Review Board. 


\section{Participants}

The sample included 66 individuals $\left(M_{\text {age }}=44.80 \pm 13.56\right.$, range $=18-65, M_{\mathrm{BMI}}=35.02 \pm 8.80$, range $=21.63-62.06$, $86.70 \%$ female, $86.40 \%$ White). Inclusion criteria were that participants (1) were 18 years of age or older; (2) had an average of at least one LOCE episode per week over the previous 12 weeks; (3) had a smartphone and were willing to complete surveys; (4) were enrolled in a treatment-based study at the Center for Weight, Eating, and Lifestyle Science and had at least 7 days before their first treatment session; and (5) were located in the United States and willing and able to participate in remote intervention and assessments. Exclusion criteria were that participants (1) were unable to fluently speak, write, and read English; (2) were planning to begin, or already participating in, another weight loss treatment or psychotherapy for LOCE in the next 16 months; (3) had a mental handicap or were experiencing other severe psychopathology that would limit their ability to engage in treatment (e.g., severe depression, substance dependence, active psychosis); and (4) were unable or unwilling to consistently complete five mobile assessments between the hours of 9 am-9 pm. All participants were administered the Eating Disorder Examination [33] and met Diagnostic and Statistical Manual of Mental Disorders [34] criteria for binge eating disorder (BED; 47.70\%, $n=31$ ), bulimia nervosa (BN; $21.50 \%, n=14$ ), other specified feeding or eating disorder (OSFED)-BED with subjectively large binge eating episodes $(12.30 \%, n=8)$, and OSFED-BN with subjectively large binge eating episodes $(18.50 \%, n=12)$.

\section{Materials}

\section{Loss of control eating}

Participants responded to the statement, "Since the last survey, have you engaged in binge eating?" on a 2-point Likert scale where no $=0$ and yes $=1$.

\section{Non-eating activities}

Participants responded to the statement, "Since the last survey, have you engaged in (chores, cooking, indoor hobby, outdoor recreation, socializing, spiritual activity/meditation, $\mathrm{TV} /$ social media, work/school, self-care/coping strategies, napping, driving, other)?" on a 2-point Likert scale where no $=0$ and yes $=1$.

\section{Negative affect}

Participants reported how much they agreed with the statement, "Right now, to what extent do you feel (guilty, sad, anxious, bored)?" on a 5-point Likert scale from 0 (not at all) to 4 (extremely). The specific NA items were selected from the Positive and Negative Affect Schedule-Expanded Form \{PANAS-X; [35]\} and their Cronbach's alpha was 0.79.

\section{Positive affect}

Participants reported how much they agreed with the statement, "Right now, to what extent do you feel (cheerful, excited, confident, attentive)?" on a 5-point Likert scale from 0 (not at all) to 4 (extremely). The specific PA items were also selected from the PANAS-X [35] and their Cronbach's alpha was 0.80 .

\section{Statistical analysis}

To maximize clarity and simplicity, the current study sought to examine NA and PA pathways separately. EMA survey responses that contained both NA and PA items above 2 (i.e., the median rating) were therefore removed for the current analyses. This data cleaning technique was chosen because previous qualitative work from our team evaluating the feasibility and acceptability of EMA to assess affective states prior to an eating episode has shown that participants reported limited understanding of why they selected high ratings of both NA and PA and attributed their selection to user error. For the preliminary aim, two generalized estimating equations (GEEs) were conducted using a logit link function and AR1 serial autocorrelation to account for the dependence within the nested data. The predictor variables (i.e., NA and PA) were centered within-person and lagged from the previous survey within the same day. For the primary aim, a series of GEEs was conducted using an identity link function and AR1 serial autocorrelation. The outcome variables (i.e., NA and PA) were centered within-person. Between-subject effects and prior LOCE episodes within the same day were controlled for. Alpha was set at 0.01 to account for multiple comparisons. Analyses were conducted using IBM SPSS 28.00.

\section{Results}

\section{EMA descriptives}

Participants completed on average $87.41 \%$ ( $\mathrm{SD}=0.14$ ) of the EMA surveys they were prompted to complete. Of the original 3432 survey sample, 301 surveys $(8.77 \%)$ were removed due to high ratings of both NA and PA. The final sample included 3,131 surveys. Participants completed on average 12 days $(\mathrm{SD}=2.43)$ of EMA recordings during the 14-day study with $159 \mathrm{~min}(\mathrm{SD}=62)$ between surveys. 


\section{Preliminary aim}

Lower than one's usual NA $(b=0.23, p=0.05)$ but not higher than one's usual PA $(b=0.04, p=0.72)$ at a given survey significantly prospectively predicted lower likelihood of a LOCE episode at the next survey.

\section{Primary aim}

GEE pathways for the primary aim are listed in Tables 1 and 2. Having recently engaged in outdoor recreation and socializing was significantly associated with lower than one's usual NA $(b=-0.14, p<0.01 ; b=-0.10, p<0.01)$ and higher than one's usual PA $(b=0.23, p<0.01 ; b=0.20$, $p<0.01)$ at the same survey, while having recently engaged in TV/social media was significantly associated with higher than one's usual NA $(b=0.08, p<0.01)$ and lower than one's usual PA $(b=-0.07, p<0.01)$. Additionally, having recently engaged in self-care/coping strategies was significantly associated with higher than one's usual PA at the same survey $(b=0.10, p<0.01)$, while having recently engaged in napping was significantly associated with lower than one's usual PA $(b=-0.07, p<0.01)$. All other pathways were non-significant.

\section{Discussion}

The purpose of the current study was to examine changes in affect from engagement in non-eating activities as predictors of LOCE using an EMA protocol. While high NA and low PA have been shown to contribute to the occurrence of LOCE, less is known about the day-to-day activities that may precede these affective experiences. Our preliminary aim was partially supported as higher than one's usual NA but not lower than one's usual PA at a given survey prospectively predicted the occurrence of a LOCE episode at the next survey. Our primary aim was supported as having
Table 1 Association between non-eating activity engagement and negative affect at the same EMA survey
Table 2 Association between non-eating activity engagement and positive affect at the same EMA survey

\begin{tabular}{lrllcc}
\hline & $\beta$ & SE & $95 \%$ CI & Wald $\chi^{2}$ & $p$ \\
\hline Chores & 0.02 & 0.02 & {$[-0.03,0.06]$} & 0.46 & 0.50 \\
Cooking & -0.01 & 0.02 & {$[-0.06,0.04]$} & 0.27 & 0.60 \\
Indoor hobby & -0.05 & 0.03 & {$[-0.10,0.01]$} & 2.75 & 0.10 \\
Outdoor recreation & -0.14 & 0.04 & {$[-0.22,-0.06]$} & 12.11 & $<0.01^{*}$ \\
Socializing & -0.10 & 0.03 & {$[-0.16,-0.04]$} & 11.09 & $<0.01^{*}$ \\
Spiritual activity/meditation & 0.03 & 0.04 & {$[-0.06,0.12]$} & 0.40 & 0.53 \\
TV/social media & 0.08 & 0.02 & {$[0.03,0.12]$} & 11.97 & $<0.01^{*}$ \\
Work/school & -0.00 & 0.02 & {$[-0.05,0.04]$} & 0.02 & 0.90 \\
Self-care/coping strategies & -0.04 & 0.04 & {$[-0.11,0.03]$} & 1.04 & 0.31 \\
Nap & 0.01 & 0.04 & {$[-0.06,0.08]$} & 0.07 & 0.79 \\
Driving & 0.04 & 0.03 & {$[-0.02,0.09]$} & 2.04 & 0.15 \\
Other & 0.04 & 0.03 & {$[-0.03,0.10]$} & 1.21 & 0.27 \\
\hline
\end{tabular}

$* p<0.01$

\begin{tabular}{lrllcc}
\hline & \multicolumn{1}{c}{$\beta$} & SE & $95 \%$ CI & Wald $\chi^{2}$ & $p$ \\
\hline Chores & 0.01 & 0.03 & {$[-0.05,0.07]$} & 0.12 & 0.74 \\
Cooking & -0.03 & 0.03 & {$[-0.09,0.04]$} & 0.76 & 0.38 \\
Indoor hobby & 0.05 & 0.05 & {$[-0.04,0.14]$} & 1.20 & 0.27 \\
Outdoor recreation & 0.23 & 0.03 & {$[0.16,0.29]$} & 44.46 & $<0.01^{*}$ \\
Socializing & 0.20 & 0.04 & {$[0.13,0.27]$} & 28.91 & $<0.01^{*}$ \\
Spiritual activity/meditation & 0.00 & 0.04 & {$[-0.09,0.09]$} & 0.00 & 0.99 \\
TV/social media & -0.07 & 0.03 & {$[-0.12,-0.02]$} & 7.51 & $<0.01^{*}$ \\
Work/school & 0.04 & 0.03 & {$[-0.02,0.09]$} & 1.84 & 0.18 \\
Self-care/coping strategies & 0.10 & 0.03 & {$[0.04,0.17]$} & 9.52 & $<0.01^{*}$ \\
Nap & -0.19 & 0.04 & {$[-0.28,-0.11]$} & 18.91 & $<0.01^{*}$ \\
Driving & 0.04 & 0.02 & {$[-0.01,0.08]$} & 2.87 & 0.09 \\
Other & -0.02 & 0.05 & {$[-0.11,0.08]$} & 0.10 & 0.75 \\
\hline
\end{tabular}

$* p<0.01$ 
recently engaged in outdoor recreation and socializing was associated with lower than one's usual NA and higher than one's usual PA at the same survey, while having recently engaged in TV/social media was associated with higher than one's usual NA and lower than one's usual PA. Additionally, having recently engaged in self-care/coping strategies was associated with higher than one's usual PA at the same survey, while having recently engaged in napping was associated with lower than one's usual PA.

Our preliminary aim finding that low PA does not prospectively predict the occurrence of LOCE is largely contrary to the existing literature; however, some studies have suggested that NA and PA might refer to different constructs [36]. Sultson, Kukk, and Akkermann [37] found that NA was more characteristic of LOCE frequency and severity than PA, suggesting that NA and PA might have different relations to LOCE. Furthermore, certain EMA studies have found that participants did not experience higher PA after engaging in LOCE and, in fact, experienced lower PA, indicating that LOCE may not serve as a maladaptive regulation strategy for PA as theorized [7, 18, 38]. These findings suggest that NA may be a more salient predictor of LOCE. Finally, previous studies have observed that men engaged in overeating more after experiencing PA than women [39, 40], which may be especially relevant for our sample that consisted mostly of women. Our finding that high NA but not low PA prospectively predicts LOCE indicates that engaging in adaptive means of NA regulation may be more effective at reducing LOCE.

Our primary aim findings suggest that individuals with LOCE experience momentary changes in affect after having recently engaged in several non-eating activities. Consistent with previous research in non-ED populations, activities such as outdoor recreation, socializing, and self-care/coping strategies appear to function as protective factors for affect, whereas TV/social media and napping appear to function as risk factors for affect. Given the association that was found between NA and LOCE, it may be beneficial for individuals to increase the frequency of activities that improve NA and thus decrease risk for LOCE. Should regulating NA in this way prove to be effective for reducing LOCE, future research may consider investigating whether this alters the relationship between PA and LOCE. For instance, PA could become a more salient predictor of LOCE once NA is regulated and stabilized, and engaging more in activities that increase PA could reduce LOCE.

\section{Strengths and limits}

Although these findings provide novel contributions to an emerging area of research, the current study was not without limitations. First, our sample included mostly White females. Future research should look to replicate these findings in a more diverse sample. Second, the use of abbreviated affect measures may have limited the extent to which NA and PA were reported. Furthermore, two of the PA items were confident and attentive, which may have been less relevant for LOCE. Third, the current study attempted to identify differential NA and PA pathways and account for user error by removing survey responses that contained high ratings of both NA and PA. Given that this may have artificially separated these affective states, future research should explore alternative data cleaning techniques. Fourth, the current study did not examine if participants were already experiencing altered NA or PA before engaging in non-eating activities. Future research should consider examining trajectories of NA and PA as opposed to tonic affect levels. Fifth, the socializing non-eating activity did not specify whether food was present, which may have inaccurately captured non-eating activity. Sixth, the current study took place during the COVID-19 pandemic, which may have altered the frequency and affective impact of certain non-eating activities. Seventh and last, the current study was underpowered to test a mediation model between non-eating activity engagement, affect, and LOCE. While the findings still support connections between the three constructs, confirming their relationship in a continuous chain would be highly valuable. Given that the current study examined associations between non-eating activity engagement, affect, and LOCE prior to treatment, future research should also seek to examine changes in these constructs over the course of treatment.

\section{What is already known on this subject?}

Engaging in LOCE in response to high NA and low PA has been widely supported as a maladaptive emotion regulation strategy $[1,5,11]$. Yet, little is known about the day-to-day activities outside of eating that may precipitate such changes in affect and thus increase or decrease risk for LOCE.

\section{What this study adds?}

The current study's findings indicate that NA may be a more salient predictor of LOCE than PA. Furthermore, a variety of day-to-day non-eating activities including outdoor recreation, socializing, TV/social media, self-care/coping strategies, and napping are associated with changes in affect and should therefore be increased or decreased to minimize risk for LOCE.

Author contributions $\mathrm{CF}$ was responsible for conceptualizing the study, conducting data analysis, and preparing the manuscript under 
the mentorship of AJ. LB, SF, and SD assisted in manuscript preparation. $\mathrm{BF}, \mathrm{AP}-\mathrm{R}$, and $\mathrm{SM}$ were responsible for data collection and preparation.

Funding No funding was received to conduct the study.

Data availability Data will be available upon request.

Code availability Software application or custom code will be available upon request.

\section{Declarations}

Conflict of interest The authors have no conflicts of interest to declare.

Ethical approval All procedures were conducted in accordance with the ethical standards of the Drexel University Institutional Review Board and with the 1964 Declaration of Helsinki and its later amendments or comparable ethical standards.

Consent to participate Informed consent was obtained from all individual participants included in the study.

Consent for publication Informed consent to publish the data collected from the study was obtained from all individual participants included in the study.

\section{References}

1. Dingemans A, Danner U, Parks M (2017) Emotion regulation in binge eating disorder: a review. Nutrients 9(11):1274-1285. https://doi.org/10.3390/nu9111274

2. Fairburn CG (2008) Cognitive behavior therapy and eating disorders. Guilford Press, New York

3. Goldschmidt AB, Wall M, Loth KA, Le Grange D, NeumarkSztainer D (2012) Which dieters are at risk for the onset of binge eating? A prospective study of adolescents and young adults. J Adolesc Health 51(1):86-92. https://doi.org/10.1016/j.jadohealth. 2011.11.001

4. Pearson CM, Zapolski TCB, Smith GT (2015) A longitudinal test of impulsivity and depression pathways to early binge eating onset. Int J Eat Disord 48(2):230-237. https://doi.org/10.1002/eat. 22277

5. Polivy J, Herman CP (1993) Etiology of binge eating: Psychological mechanisms. In: Fairburn CG, Wilson GT (eds) Binge eating: nature, assessment, and treatment. Guilford Press, New York, pp 173-205

6. Wardle J (1985) The binge-purge syndrome: diagnosis, treatment and research. Behav Res Ther 23(5):620. https://doi.org/10.1016/ 0005-7967(85)90128-7

7. Haedt-Matt AA, Keel PK (2011) Revisiting the affect regulation model of binge eating: a meta-analysis of studies using ecological momentary assessment. Psychol Bull 137(4):660-681. https://doi. org/10.1037/a0023660

8. Berg KC, Crosby RD, Cao L, Peterson CB, Engel SG, Mitchell JE, Wonderlich SA (2013) Facets of negative affect prior to and following binge-only, purge-only, and binge/purge events in women with bulimia nervosa. J Abnorm Psychol 122(1):111-118. https:// doi.org/10.1037/a0029703

9. Schaefer LM, Smith KE, Anderson LM, Cao L, Crosby RD, Engel SG, Wonderlich SA (2020) The role of affect in the maintenance of binge-eating disorder: evidence from an ecological momentary assessment study. J Abnorm Psychol 129(4):387-396. https://doi. org/10.1037/abn0000517

10. Smyth JM, Wonderlich SA, Heron KE, Sliwinski MJ, Crosby RD, Mitchell JE, Engel SG (2007) Daily and momentary mood and stress are associated with binge eating and vomiting in bulimia nervosa patients in the natural environment. J Consult Clin Psychol 75(4):629-638. https://doi.org/10.1037/0022-006X.75.4.629

11. Mason TB, Smith KE, Anderson LM, Hazzard VM (2021) Anhedonia, positive affect dysregulation, and risk and maintenance of binge-eating disorder. Int J Eat Disord 54(3):287-292. https://doi. org/10.1002/eat.23433

12. Small DM, Jones-Gotman M, Dagher A (2003) Feeding-induced dopamine release in dorsal striatum correlates with meal pleasantness ratings in healthy human volunteers. Neuroimage 19(4):1709-1715. https://doi.org/10.1016/S1053-8119(03) 00253-2

13. Munsch S, Meyer AH, Quartier V, Wilhelm FH (2011) Binge eating in binge eating disorder: a breakdown of emotion regulatory process? Psychiatry Res 195(3):118-124. https://doi.org/10. 1016/j.psychres.2011.07.016

14. Wonderlich JA, Breithaupt L, Thompson JC, Crosby RD, Engel SG, Fischer S (2018) The impact of neural responses to food cues following stress on trajectories of negative and positive affect and binge eating in daily life. J Psychiatr Res 102:14-22. https://doi. org/10.1016/j.jpsychires.2018.03.005

15. Linardon J (2018) Rates of abstinence following psychological or behavioral treatments for binge-eating disorder: meta-analysis. Int J Eat Disord 51(8):785-797. https://doi.org/10.1002/eat.22897

16. Linardon J, Messer M, Fuller-Tyszkiewicz M (2018) Meta-analysis of the effects of cognitive-behavioral therapy for binge-eatingtype disorders on abstinence rates in nonrandomized effectiveness studies: comparable outcomes to randomized, controlled trials? Int J Eat Disord 51(12):1303-1311. https://doi.org/10.1002/eat. 22986

17. The Diabetes Prevention Program Research Group (2002) The diabetes prevention program (DPP): description of lifestyle intervention. Diabetes Care 25(12):2165-2171. https://doi.org/10.2337/ diacare.25.12.2165

18. Stein RI, Kenardy J, Wiseman CV, Dounchis JZ, Arnow BA, Wilfley DE (2007) What's driving the binge in binge eating disorder?: a prospective examination of precursors and consequences. Int $\mathrm{J}$ Eat Disord 40(3):195-203. https://doi.org/10.1002/eat.20352

19. Smith KE, Mason TB, Juarascio A, Weinbach N, Dvorak R, Crosby RD, Wonderlich SA (2020) The momentary interplay of affect, attention bias, and expectancies as predictors of binge eating in the natural environment. Int J Eat Disord 53(4):586-594. https://doi.org/10.1002/eat.23235

20. Mosko JE, Delach MJ (2021) Cooking, creativity, and well-being: an integration of quantitative and qualitative methods. J Creat Behav 55(2):348-361. https://doi.org/10.1002/jocb.459

21. Kuykendall L, Lei X, Zhu Z, Hu X (2020) Leisure choices and employee well-being: comparing need fulfillment and well-being during TV and other leisure activities. Appl Psychol Health Well Being 12(2):532-558. https://doi.org/10.1111/aphw.12196

22. Jenkins AR (2015) Digital gamers: a mixed method study of players, emotion, mood, and moral life. (Doctoral dissertation). Retrieved from ProQuest Dissertations \& Theses Global. (3714014)

23. Burns AC, Saxena R, Vetter C, Phillips AJK, Lane JM, Cain SW (2021) Time spent in outdoor light is associated with mood, sleep, and circadian rhythm-related outcomes: a cross-sectional and longitudinal study in over 400,000 UK Biobank participants. J Affect Disord 295:347-352. https://doi.org/10.1016/j.jad.2021.08.056

24. Kondo MC, Triguero-Mas M, Donaire-Gonzalez D, Seto E, Valentín A, Hurst G, Nieuwenhuijsen MJ (2020) Momentary mood response to natural outdoor environments in four European 
cities. Environ Int 134:105237. https://doi.org/10.1016/j.envint. 2019.105237

25. Chesbro GA, Peterson JA, Black CD, Larson DJ, Larson RD (2021) The effect of social distancing during COVID-19 on psychological mood and physical activity. Med Sci Sports Exerc 53(8):343-343. https://doi.org/10.1249/01.mss.0000763200. 89203.59

26. Jones DR, Graham-Engeland JE, Smyth JM, Lehman BJ (2018) Clarifying the associations between mindfulness meditation and emotion: daily high- and low-arousal emotions and emotional variability. Appl Psychol Health Well Being 10(3):504-523. https:// doi.org/10.1111/aphw.12135

27. Sirotina U, Shchebetenko S (2020) Loving-kindness meditation and compassion meditation: do they affect emotions in a different way? Mindfulness 11(11):2519-2530. https://doi.org/10.1007/ s12671-020-01465-9

28. Bernstein JPK, Calamia M (2019) Dimensions of driving-related emotions and behaviors: an exploratory factor analysis of common self-report measures. Accid Anal Prev 124:85-91. https://doi.org/ 10.1016/j.aap.2019.01.004

29. Ru T, Chen Q, You J, Zhou G (2019) Effects of a short midday nap on habitual nappers' alertness, mood and mental performance across cognitive domains. J Sleep Res 28(3):1-11. https://doi.org/ $10.1111 /$ jsr. 12638

30. Kaida K, Takahashi M, Otsuka Y (2007) A short nap and natural bright light exposure improve positive mood status. Ind Health 45(2):301-308. https://doi.org/10.2486/indhealth.45.301

31. Goldschmidt AB, Wonderlich SA, Crosby RD, Engel SG, Lavender JM, Peterson CB, Mitchell JE (2014) Ecological momentary assessment of stressful events and negative affect in bulimia nervosa. J Consult Clin Psychol 82(1):30-39. https://doi.org/10.1037/ a0034974

32. Srivastava P, Michael ML, Manasse SM, Juarascio AS (2021) Do momentary changes in body dissatisfaction predict binge eating episodes? An ecological momentary assessment study. Eat Weight Disord 26(1):395-400. https://doi.org/10.1007/ s40519-020-00849-z
33. Cooper Z, Fairburn C (1987) The eating disorder examination: a semi-structured interview for the assessment of the specific psychopathology of eating disorders. Int J Eat Disord 6(1):1-8. https://doi.org/10.1002/1098-108x(198701)6:1\%3c1::Aid-eat22 60060102\%3e3.0.Co;2-9

34. American Psychiatric Association (2013) Diagnostic and statistical manual of mental disorders, 5th edn. Author, Arlington

35. Watson D, Clark LA (1999) The PANAS-X: manual for the positive and negative affect schedule — expanded form. University of Iowa, Iowa. https://doi.org/10.17077/48vt-m4t2

36. van Strien T, Cebolla A, Etchemendy E, Gutiérrez-Maldonado J, Ferrer-García M, Botella C, Baños R (2013) Emotional eating and food intake after sadness and joy. Appetite 66:20-25. https://doi. org/10.1016/j.appet.2013.02.016

37. Sultson H, Kukk K, Akkermann K (2017) Positive and negative emotional eating have different associations with overeating and binge eating: construction and validation of the positive-negative emotional eating scale. Appetite 116:423-430. https://doi.org/10. 1016/j.appet.2017.05.035

38. Wegner KE, Smyth JM, Crosby RD, Wittrock D, Wonderlich SA, Mitchell JE (2002) An evaluation of the relationship between mood and binge eating in the natural environment using ecological momentary assessment. Int J Eat Disord 32(3):352-361. https:// doi.org/10.1002/eat.10086

39. Macht M, Roth S, Ellgring H (2002) Chocolate eating in healthy men during experimentally induced sadness and joy. Appetite 39(2):147-158. https://doi.org/10.1006/appe.2002.0499

40. Nolan LJ, Halperin LB, Geliebter A (2010) Emotional appetite questionnaire. Construct validity and relationship with BMI. Appetite 54(2):314-319. https://doi.org/10.1016/j.appet.2009. 12.004

Publisher's Note Springer Nature remains neutral with regard to jurisdictional claims in published maps and institutional affiliations. 